\title{
The Effect of Gold Nanoparticle on Absorbed Dose by IMRT in Esophagus Phantom
}

\author{
Zeinabnamazi Shiraazi', Seyedrabie Mahdavi², Mohammad Mahdavi1, \\ Mahboubeh Khadem Abolfazli ${ }^{*}$ \\ ${ }^{1}$ Physics Department, Mazandaran University, Babolsar, Iran \\ ${ }^{2}$ Medical Physics Department, Iran University of Medical Science, Tehran, Iran \\ ${ }^{3}$ Radiation Department, Babol University of Medical Science, Babol, Iran \\ Email: ${ }^{*}$ Mahboubeh.khadem@yahoo.com
}

Received 5 September 2015; accepted 23 September 2015; published 29 September 2015

Copyright (C) 2015 by authors and OALib.

This work is licensed under the Creative Commons Attribution International License (CC BY).

http://creativecommons.org/licenses/by/4.0/

(c) (i) Open Access

\section{Abstract}

This study aimed to evaluate the use of dosimeters MAGICA gel impregnated with nanoparticles of gold to increase the dose of faulty tissue in the chest phantom in the esophagus and other organs to determine the dose and dose distribution in the tissue with an IMRT treatment. In this study, based on previous studies, the optimal concentration of $0.1 \mathrm{mM}$ gold nanoparticles has been considered. Also, better localization and avoid radiation received dose by other organizations around the IMRT machine used for radiation. For comparison and understanding of dose distribution and dose rate increase factor (DEF), gel dosimeters were acquired by MRI. The results showed that by adding gold nanoparticles with a diameter of $50 \mathrm{~nm}$ and optimum concentration is $0.1 \mathrm{mM}$, the dose and energy $500 \mathrm{cGy}$ and $6 \mathrm{MV}$ with IMRT respectively, the factors increasing the dose (DEF) is about $9.74 \%$. It's remarkable, this research is that Esophagus phantom to containing gold nanoparticles to other tissues (lung and spinal cord) and also the Phantom of the esophagus in the absence of gold nanoparticles, has received the highest dose and compared with the non-nano $R_{2}$ Increased. It is also a good match between the results of dosimetry gel and results of treatment.

\section{Keywords}

IMRT, Dosimetry, Gold Nanoparticle, Esophagus Phantom

Subject Areas: Biophysics

\footnotetext{
${ }^{*}$ Corresponding author.
}

How to cite this paper: Shiraazi, Z., Mahdavi, S., Mahdavi, M. and Abolfazli, M.K. (2015) The Effect of Gold Nanoparticle on Absorbed Dose by IMRT in Esophagus Phantom. Open Access Library Journal, 2: e1919. 


\section{Introduction}

The greatest challenge for radiation therapy or any cancer therapy is to attain the highest probability of cure with the least morbidity. The simplest way in theory to increase this therapeutic ratio with radiation is to encompass all cancer cells with sufficient doses of radiation during each fraction, while simultaneously sparing surrounding normal tissues. In practice, however, we have been hampered by our abilities to both identify the cancer cells and target them with radiation. Over the past decade, enormous progress has been made on both fronts. Technical improvements in the application of X-rays, computed tomography scans, magnetic resonance imaging with and without spectroscopy, ultrasound, positron emission tomography scans, and electronic portal imaging —and our understanding of their limitations - have greatly improved our ability to identify tumors. Intensity-modulated radiation therapy (IMRT) can sculpt the high-dose volume around the site of disease with hitherto unachievable precision. Conformal avoidance of normal tissues goes hand in hand with this. Inhomogeneous dose painting is possible. It is important that we know the difference between normal tissue and tumor tissue for the development of hybrid nanoparticles for the diagnosis and treatment of cancer. Normal tissue with vessel walls, narrow and continuous pores is often about $9 \mathrm{~nm}$ and rarely about $50 \mathrm{~nm}$. Thus, small molecules with molecules such as polymers which are very slow in the context of their influence, they easily enter the tissue. However, tumor tissue, rich organ such as liver, spleen and bone marrow with discontinuous capillary walls and a large number of pores are about $100 \mathrm{~nm}$. In addition, the capillary walls do not batch basic broadband to allow particles less than $100 \mathrm{~nm}$ so easily [1]. As this phenomenon is explained in detail in Ref. [1]. Interestingly no tumor tissue-polymeric material and lipophilic lymphatic system eliminate them [2]. Thus, when the particles penetrate the tumor tissue, they are easily wiped. Accordingly, the influence of increased survival due to particles 50 - 10 nm shows [3].

50 - $100 \mathrm{~nm}$ medium-sized gold nanoparticles to target and treat tumors are suitable for imaging purposes. Kunar and colleagues have shown that gold nanoparticles into the cells, even though they are non-toxic to human cells. However, some of the precursors used to produce nanoparticles may be toxic [4]. It is important for the toxicity of gold nanoparticles that can use non-toxic reagents for production control. In this study we use polymer gel phantom with gold nanoparticle irradiate by IMRT, because our aim is to achieve the best absorbed dose enhancement with the effect of gold nanoparticle and IMRT.

\section{Ease of Use}

\subsection{Gel Fabrication}

This study made of Plexiglas Glass Esophagus phantom and gel polymer impregnated with gold nanoparticles and is sensitive to radiation of MAGICA done. The results of treatment in this study intended as a reference design. Experiments on dosimetry in an energy gel repeated four times. The gel used in the study of polymer is abbreviated MAGICA. Gel method is similar to the method described by Fang et al. (2001) [5]. Materials and quantities required for the production of 750gr of gel in Table 1 is.

Gold nanoparticles used in this study have a diameter of nm50 concentration ppm 250 or $0.25 \mathrm{mg} / \mathrm{ml}$ that the company was prepared nano Zino.

When the MAGICA gel was fabricated, GN-MAGICA gel, amounting to $141.3 \mathrm{ml}$ MAGICA gel separate the gold nanoparticles and then was placed in a separate container. The containers containing $141.3 \mathrm{ml}$ of gel MAGICA were placed on the mixer. The gold nanoparticles with $0.1 \mathrm{mM}$ concentration were added and the gel was mixed mechanically with gold nanoparticles and then after 5 minutes GN-MAGICA gel is made. $150 \mathrm{ml}$ of gel MAGICA within ten, $15 \mathrm{ml}$ Falcon tube gel were cast for calibration. The rest of the Phantom MAGICA cylindrical gel phantom Esophagus four, three phantom lungs into the spinal cord (Figure 3 and Figure 4). GNMAGICA amount of gel that was prepared by Esophagus moved into a cylindrical phantom. Gels and GNMAGICA MAGICA of each sample in the same cylinder volume is a control sample that shown Figure 1.

\subsection{Irradiation Gel}

Phantom, 24 hours after irradiation is to the construction phase of Pars Hospital (Figure 2). IMRT devices (manufactured by Siemens) were used for the irradiation. Before irradiation run gels by treatment planning system on the Core Plan. Irradiation Phantom of the esophagus, lung and spinal cord in the chest phantom was placed on three angles of 0,120 and 240 degrees give $6 \mathrm{MV}$ energy. IMRT radiation particle has shown in 


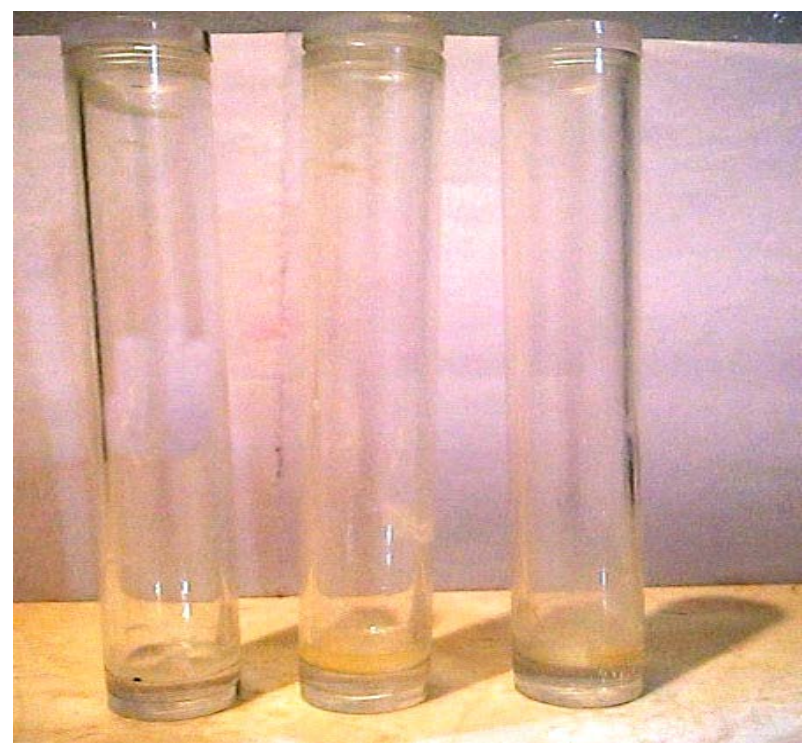

Figure 1. Cylindrical gel phantom esophagus.

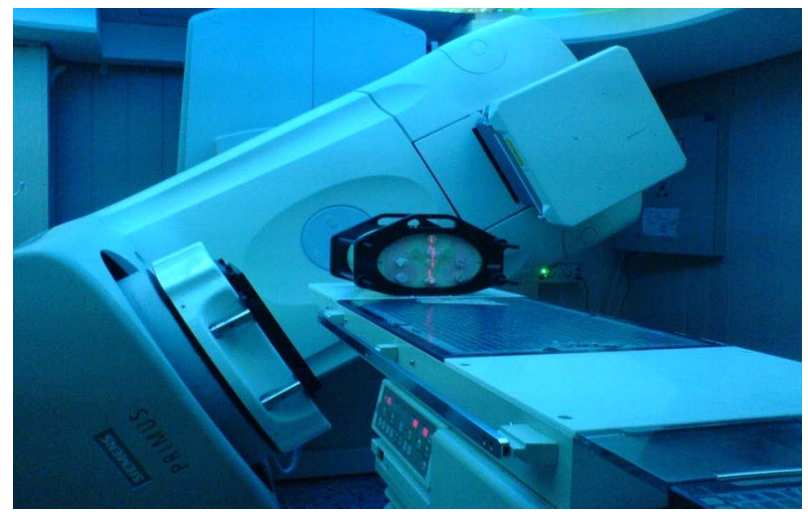

Figure 2. View of the phantom chest radiation by IMRT.

Table 1. Materials and quantities necessary to 750 gr of gel MAGICA.

\begin{tabular}{cccc}
\hline Component & Calculated amount (gr) & Actual amount (gr) & Company of product \\
\hline Agarose & 3.7500 & 3.7526 & Merc \\
Gelatin & 60 & 60.0029 & Merc \\
Methacrylic acid & 67.5000 & 67.5000 & Merc \\
$\mathrm{CuSO}_{4} \cdot 5 \mathrm{H}_{2} \mathrm{O}$ & 0.0112 & 0.1125 & Merc \\
Hydroquinone & 1.5000 & 1.5009 & Merc \\
Ascorbic acid & 0.2638 & 0.2638 & Merc \\
Water (distilled water) & $616.4500(\mathrm{ml})$ & $616.5000(\mathrm{ml})$ & Zolaliran \\
\hline
\end{tabular}

Table 2. Phantom of the esophagus, lung and spinal cord of the chest were MAGICA and only the fourth time Esophagus gel containing no nanoparticles were used in the Phantom. The four irradiated with angles and energy was mentioned above. Esophagus phantom containing a sample and a sample containing GN-MAGICA MAGICA without irradiation remain as a control. It is noteworthy that the Phantom and Phantom Esophagus as cancerous tissue is considered as healthy tissue around the lung and spinal cord (OAR) will be examined. 


\subsection{MRI Imaging}

Imaging phantom containing gel GN-MAGICA and MAGICA Between one day after irradiation was performed, this time for all the monomers in the polymer gel, is to reduce the error and fixing the radiation gel polymerization process in considered. For imaging, the field strength of 1.5 Tesla MRI machine made in Siemens with Bahman hospital with head coil was used. Spin-echo imaging in multi-protocol 16 echo number listed in Table 3 was performed. The shooting lasted 30 minutes.

\subsection{Image Processing}

All images were transferred to a computer for processing basic DICOM format. $\mathrm{R}^{2}$ distribution is calculated using base image was 16 . First the images *.dcm changed. The star here is the four digit number to the image. The maps $R^{2}$ by using a software program computing environment Matlab (MATLABTM, Version R2012b, Math works) were calculated.

\section{Result}

After reading tubing Falcon radiation with IMRT technique in the dose range 0 - 600 cGy, by MRI with image analysis, using computational software MATLAB, the mean and standard deviation (SD) $\mathrm{R}^{2}$ for each dose specific is measured. The spin-spin relaxation time track gel dose response curve of hydrogen $\left(R^{2}=1 / T^{2}\right)$ is drawn in terms of dose (Figure 3). As we placed the gel dosimeters behavior 0 - $600 \mathrm{cGy}$ dose range is quite linear $\left(\mathrm{r}^{2}\right.$ $=0.997)$. Slope and sensitivity to dose in the range of $0.003(\text { s.cGy })^{-1}$ and the value $\left(\mathrm{R}^{2}\right)_{0}$ is $4.199(\mathrm{~s})^{-1}$.

\subsection{Dose Enhancement Factor}

According to the maps obtained $\mathrm{R}^{2}$, the factor that ratio dose response increase dose to dose response Gel GN-MAGICA that both are exposed to concentrations $0.1 \mathrm{mM}$ amount DEF 9.74\%, respectively, which match well with other studies.

Isodose curves for Phantom radiates by the MATLAB computational software were obtained.

According to Figures 4-6 that are seen away from the central axis during each cylindrical phantom, the percentage dose decreased.

\subsection{The Result of Treatment Planning}

Before irradiation to the gel, a three-dimensional scan of the chest phantom was prepared using an inverse design program Core Plan was prepared for treatment. So that all orders including the angle, the number of rays, radiation, how the MLC, the dose should receive Esophagus as the object enters or dose distribution curve isodoses in Esophagus also anticipated. This treatment has been designed to findings of the season after the results

Table 2. IMRT radiation protocol phantom.

\begin{tabular}{cccc}
\hline Beam name & Beam 1 & Beam 2 & Beam 3 \\
\hline Radiation energy & $6 \mathrm{MV}$ & $6 \mathrm{MV}$ & 84.56 \\
Setup SSD $(\mathrm{cm})$ & 93.03 & 84.08 & 240 \\
Gantry angle & 0 & 120 & $74 \mathrm{MU}$ \\
Time/MU & $74 \mathrm{MU}$ & $73 \mathrm{MU}$ & \\
\hline
\end{tabular}

Table 3. MRI used protocol.

\begin{tabular}{ccc} 
TR & $3250 \mathrm{~ms}$ \\
TE & $22-352 \mathrm{~ms}$ \\
No. echo & 16 \\
Matrix size & $192 \times 256 \mathrm{~mm}^{2}$ \\
\hline
\end{tabular}




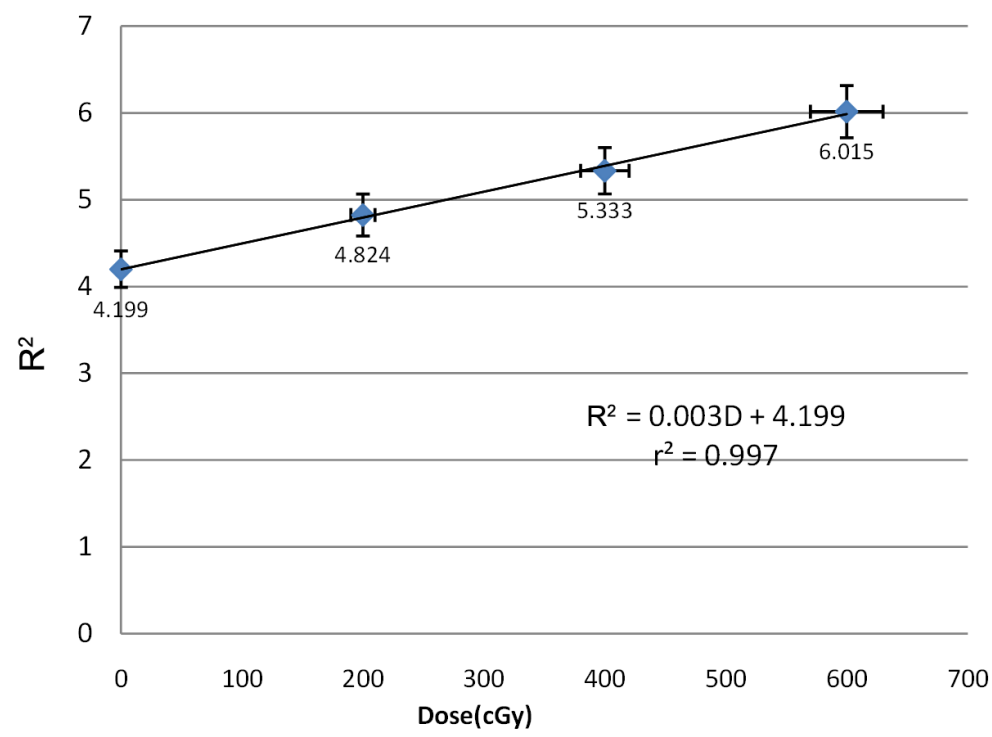

Figure 3. Curve dose of gel calibrated MAGICA.
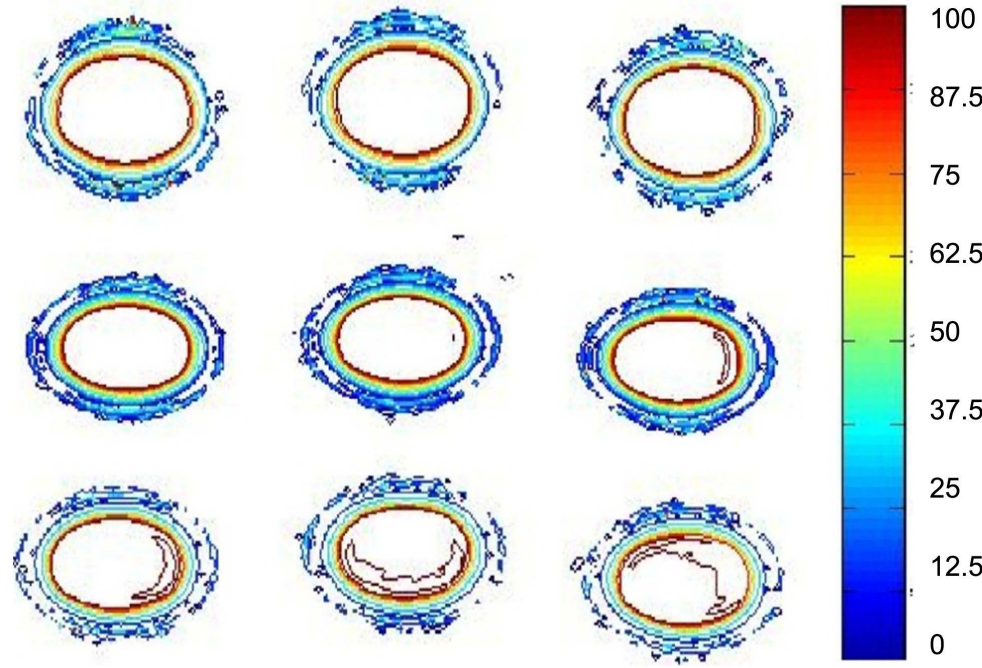

25

Figure 4. Isodose curves for phantom radiates by the MATLAB software in first transects for cross-section view.

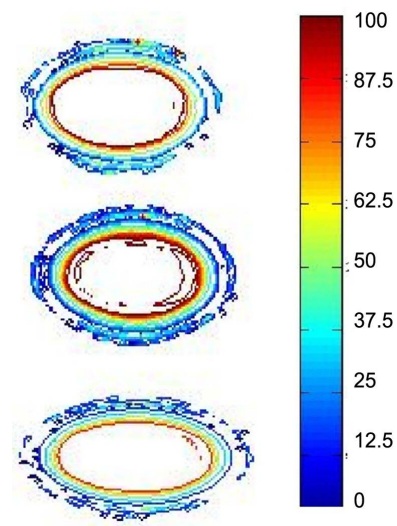

Figure 5. Isodose curves obtained in third transects for cross-section view. 

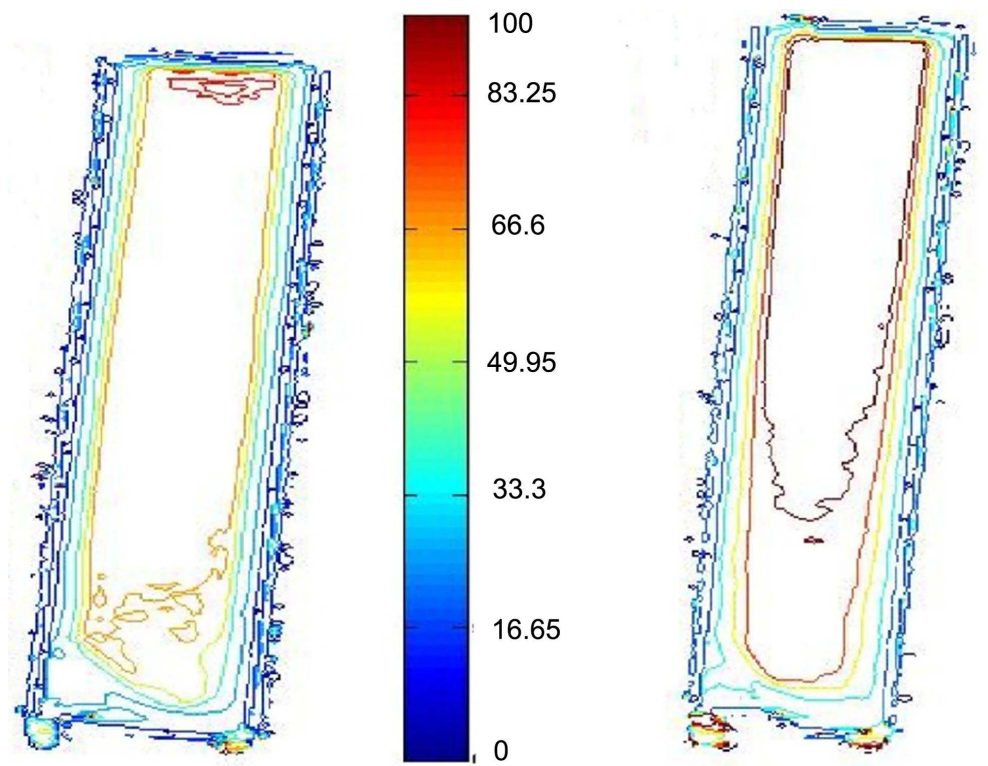

Figure 6. Isodose-curves obtained in the two horizontal axes cutting in for forth transects.

of laboratory work and design treatment will be compared with each other.

As the reverse of the Figure 7 is shown the central axis, through the esophagus will receive the highest percentage of dose. The values of the delivered dose and curves isodoses with color separation respectively in the two cross and Esophagus in the central organs (7) is shown.

\subsection{Dose Volume Histogram (DVH)}

Dose-volume histograms are a view of the way, dose-volume publication. As you see in Figure 8, the esophagus as the target radiation, lung and spine of each volume ratio to receive the highest and lowest dose.

\subsection{Compare of the Isodose Curves}

According to isodose curves, and esophagus with GN-MAGICA and MAGICA a good match with isodose curves in the design of treatment. Note that phantom esophagus with gold nanoparticles because of their high sensitivity to radiation absorption curves in the design of treatment isodose closer.

By comparing the profiles Dose treatment planning, Esophagus of nanoparticles and Esophagus without nanoparticles in both horizontal and vertical axes, can get the relative percentage of absorbed dose in the phantom esophagus GN-MAGICA because of the contrast agent causing gold nanoparticles corresponded very closely to Profile design treatment.

According to the Figures 9-13, we can see that the design of the reverse map dosimetry verification of the authenticity of the gel. Also in forms (12) and (13) is shown that the fact of the radiation field surrounding the target volume away from the center of the target volume is reduced relative dose rates.

\section{Discussion}

One of the main goals of adaptive radiation, defective tissue and yet maintain delivery of the highest dose to surrounding healthy tissue from radiation. A lot of work has been done in this regard, one of the ways to solve this problem, the use of materials like metal or metal with a high atomic number to increase the dose of a cancer lump. The first of these elements such as iodine and gadolinium were using, but because it was difficult for kidney disease should be excluded. The high atomic number of an element that can have texture and durability with a gold element is not toxic to cells; this element in addition to the benefits of increased cross-section facing incoming rays will also deal in. It was seen in the dose range $600-0 \mathrm{cGy}, \mathrm{R}^{2}$ Dose graph graphing linear with a slope of the sensitivity of the dose was 0.003 (s.cGy) ${ }^{-1}$. Phantom Esophagus also dipped in gold nanoparticles, a 


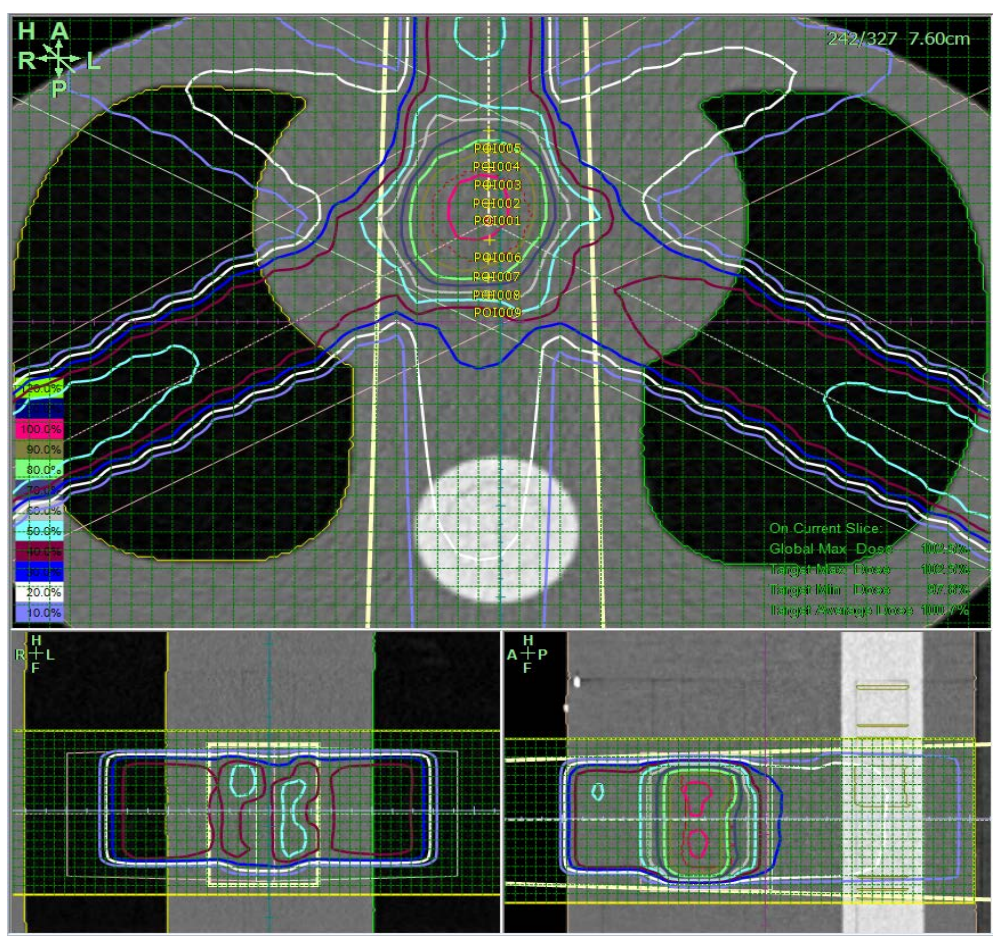

Figure 7. View of treatment planning of the program core plan centered on the cross and esophagus.

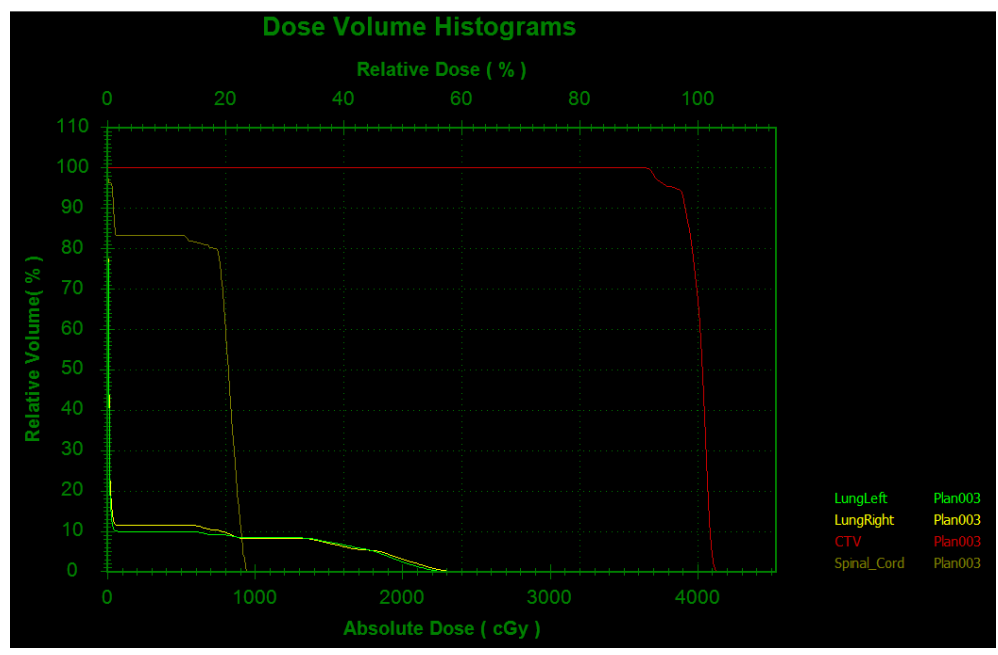

Figure 8. Dose-volume histograms view.

higher dose of radiation than the Phantom Esophagus received no gold nanoparticles and the dose enhancement factor (DEF) by about $9.740 \%$, respectively, whiles the surrounding healthy tissues such as the lungs and spinal cord without nanoparticles Gold received the highest dose. Also call $\mathrm{R}^{2}$ in MAGICA polymer gel was irradiated beam of radiation compared to the 5.7 percent increase given that this represents a good polymerization process. Also polymer gel dosimetry approved results of experimental work and the results of the design of a good overlap with each other.

Dosimeter gels are as a chemical dosimeter capable of measuring the dose distribution in three dimensions. The dosimeter uses gold nanoparticles to increase the amount of DEF so according to the results of the treatment beam gold nanoparticles can be used as a boost dose of radiation, the radiation dosimeter with IMRT technique for localized radiation is operated in such a way that the maximum dose of cancerous tissue and surrounding 


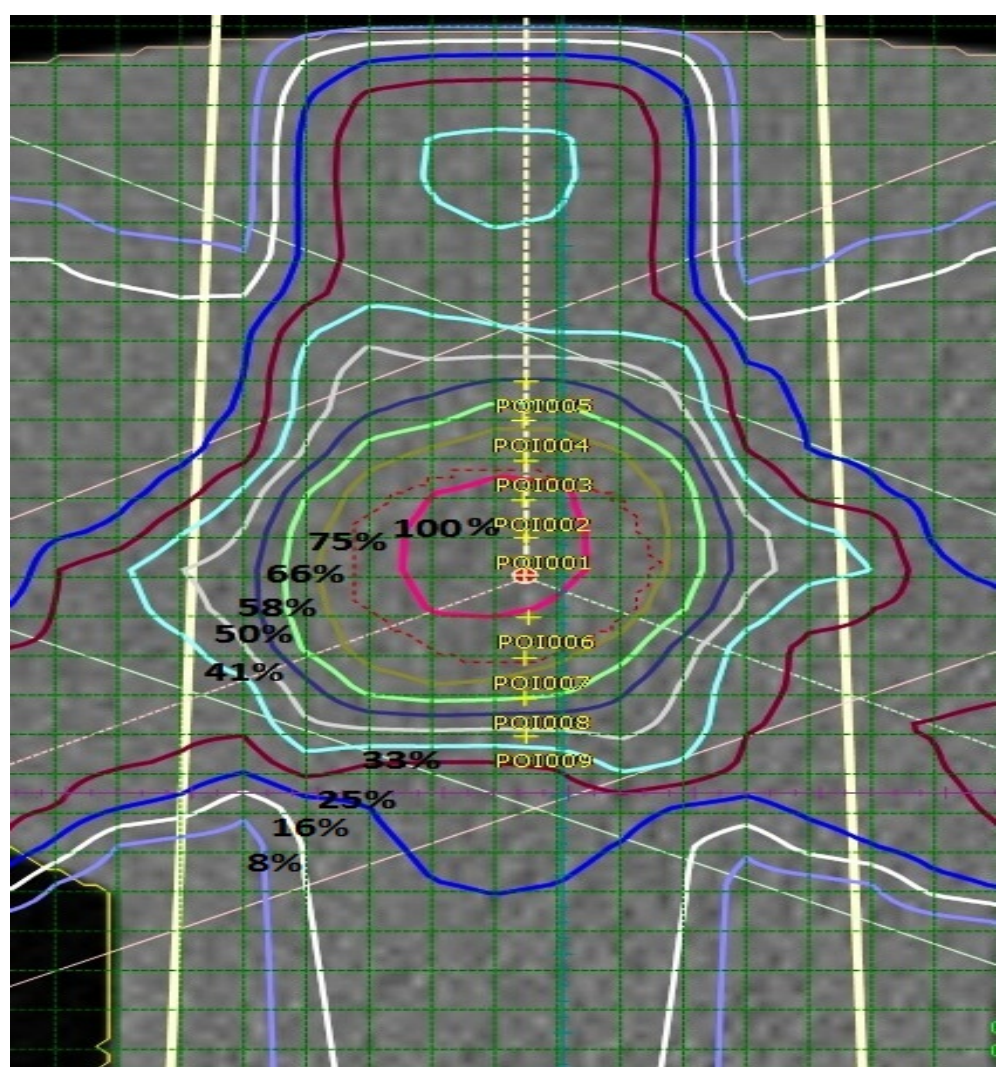

Figure 9. Percent of the dose delivered to the esophagus in the treatment planning of the isodosecurves.

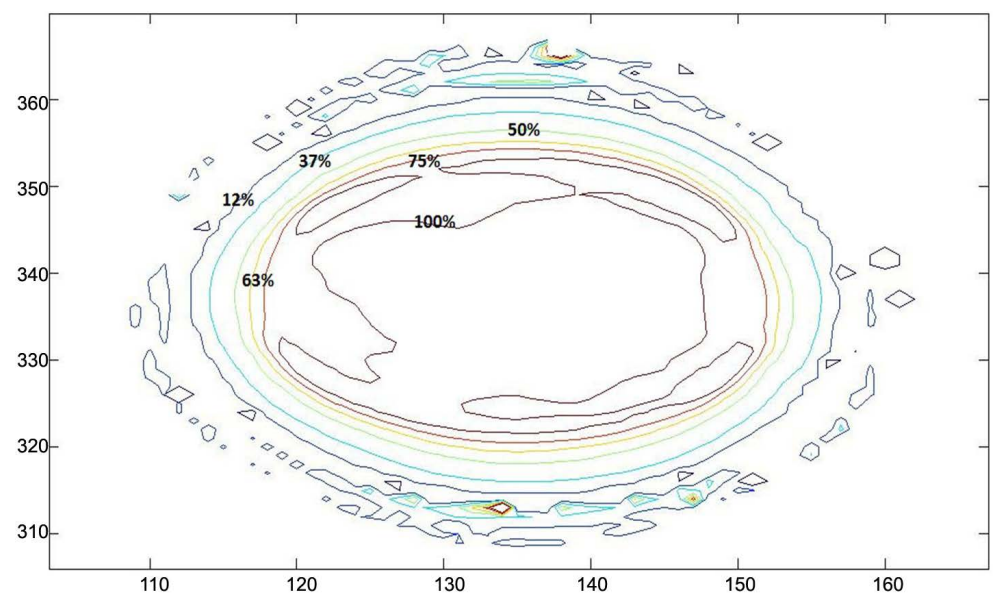

Figure 10. Phantom dose of esophagus with GN-MAGICA plan isodose.

normal tissues receive the least amount of dosage. Moreover, the results obtained by treatment planning, will be confirmed. Also khadem et al. achieved $10 \%$ absorption dose enhancement with $0.1 \mathrm{mM}$ concentration gold nanoparticle in MAGICA polymer $18 \mathrm{MV}$ energy Linear accelerator [6]. In other research Mahdavi et al. studied effect of gold nanoparticle on percentage depth dose enhancement on megavoltage energy in MAGICA polymer gel dosimeter. Experimental results have shown depth dose increase of $10 \%, 2 \%$ and $4 \%$ in $0.1 \mathrm{mM}$, $0.2 \mathrm{mM}$ and $0.4 \mathrm{mM}$ concentrations, respectively [7]. Ataei et al. compare dose enhancement factor of $6 \mathrm{MV}$ and $18 \mathrm{MV}$ in $0.1 \mathrm{mM}$, concentration gold nanoparticle in MAGICA polymer gel. The results showed that by adding of gold nanoparticles to the MAGICA polymer gel absorbed dose is increased. The levels of polymerization of 


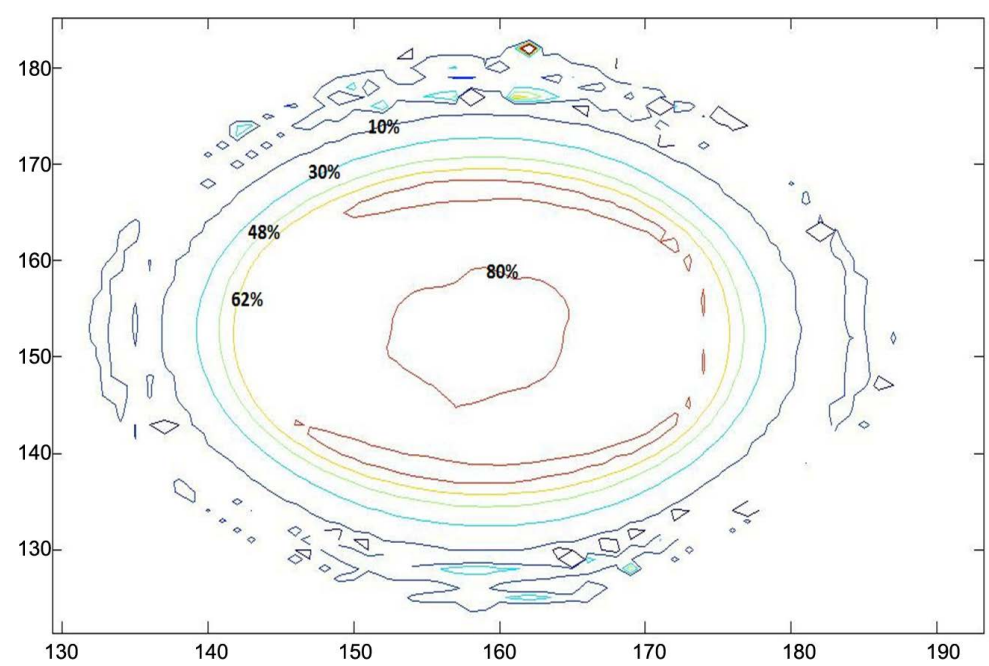

Figure 11. Phantom dose of esophagus contains MAGICA plan isodose.

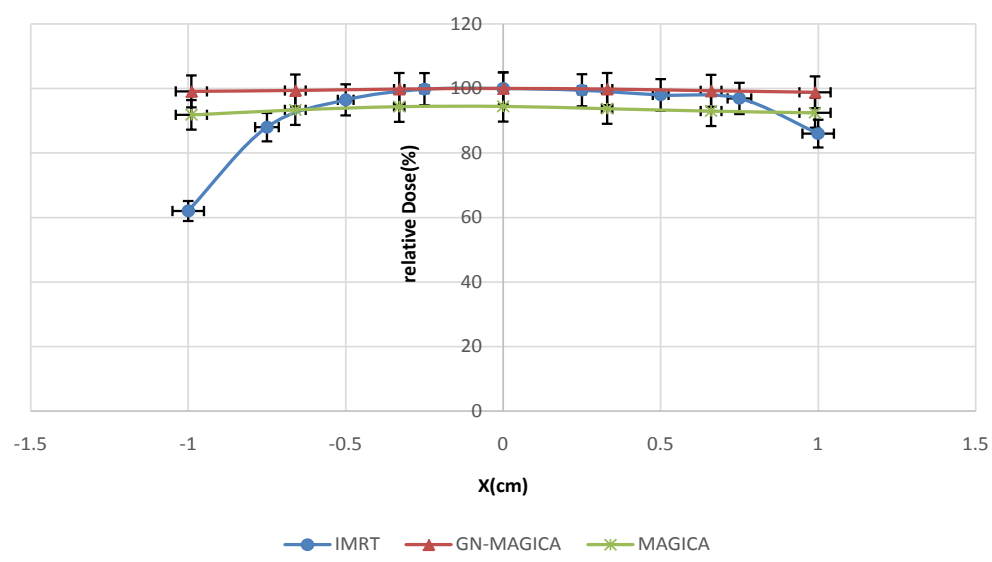

Figure 12. Dose profile along the horizontal axis in the esophagus.

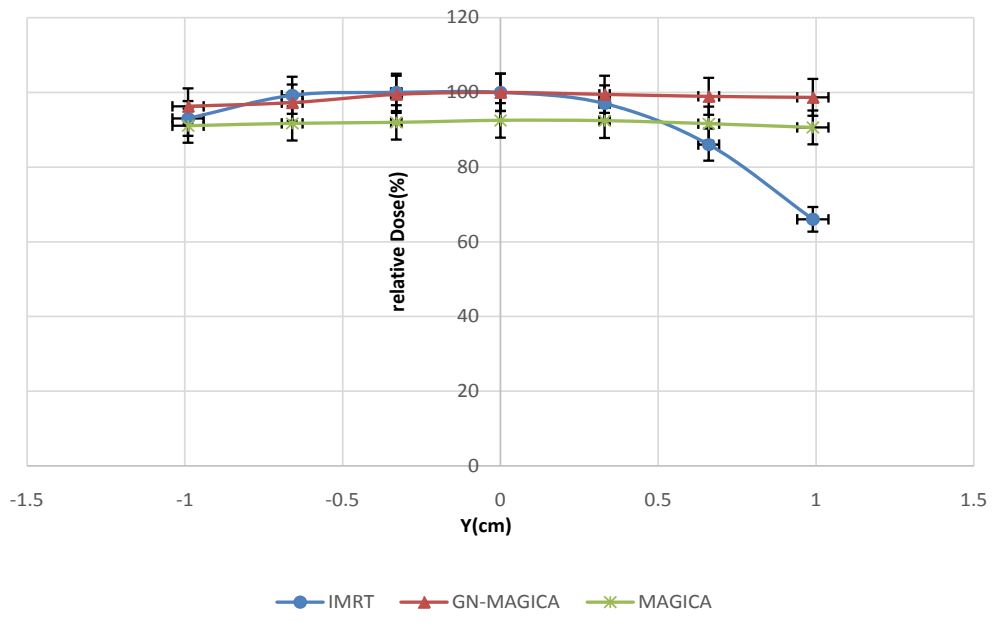

Figure 13. Dose profile along the vertical axis in the esophagus.

irradiated gels with and without AuNPs in energy $6 \mathrm{MV}$ is more than energy $18 \mathrm{MV}$. It seems that because of the dominance of photoelectric effect at low energies and pair production effect at high energies [8]. The re- 
search of gel polymer impregnated with gold nanoparticles by the optimum concentration $0.1 \mathrm{mM}$ MAGICA type and size of mega-voltage beams $50 \mathrm{~nm}$ to increase the dose in the phantom Esophagus $6 \mathrm{MV}$ energy is used.

\section{Conclusion}

In this study, in order to enhance this important goal radiotherapy comparative mentioned above for radiation, IMRT was used for this method compared to other methods of treatment for localized operated and would deliver its maximum dose to cancerous tissue and the lowest dose to surrounding healthy organs .

\section{Acknowledgements}

Thanks of Bahman and Pars hospital staff for cooperation of MRI and IMRT.

\section{References}

[1] Letsinger, R.L., Mirkin, C.A., Elghanian, R., Mucic, R.C. and Storhoff, J.J. (1999) Chemistry of Oligonucleotide-Gold Nanoparticle Conjugates. Phosphorus, Sulfur and Silicon and the Related Elements, 144, 359-362. http://dx.doi.org/10.1080/10426509908546255

[2] Maeda, H., Seymour, L.W. and Miyamoto, Y. (1992) Conjugates of Anticancsr Agents and Polymers-Advantages of Macromolecular Therapeutics in Vivo. Bioconjugate Chemistry, 3, 351-362. http://dx.doi.org/10.1021/bc00017a001

[3] Qi, X.R., Maitani, Y., Nagai, T. and Wei, S.L. (1997) Comparative Pharmacokinetics and Antitumor Efficacy of Doxorubicin Encapsulated in Soybean-Derived Sterols and Poly (Ethylene Glycol) Liposomes in Mice. International Journal of Pharmaceutics, 146, 31-39. http://dx.doi.org/10.1016/S0378-5173(96)04753-9

[4] Connor, E.E., Mwamuka, J., Gole, A., Murphy, C.J. and Wyatt, M.D. (2005) Gold Nanoparticles Are Taken up by Human Cells but Do Not Cause Cytotoxicity. Small, 1, 325-327. http://dx.doi.org/10.1002/smll.200400093

[5] Fong, P.M., Kiel, D.Z., Does, M.D. and Core, J.C. (2001) Polymer Gels for Magnetic Resonance Imaging of Radiation Dose Distributions at Normal Room Atmosphere. Physics in Medicine and Biology, 46, 3105-3113. http://dx.doi.org/10.1088/0031-9155/46/12/303

[6] Khadem Abolfazli, M., Mahdavi, M., Mahdavi, S.R.M. and Ataei, Gh. (2013) Dose Enhancement Effect of Gold Nanoparticles on MAGICA Polymer Gel in Mega Voltage Radiation Therapy. International Journal of Radiation Research, 11, 55-61.

[7] Mahdavi, M., Khadem Abolfazli, M., Mahdavi, S.R.M. and Ataei, Gh. (2013) Effect of Gold Nanoparticle on Percentage Depth Dose Enhancement on Megavoltage Energy in MAGICA Polymer Gel Dosimeter. Journal of Biomedical Physics and Engineering, 3, 37-44.

[8] Atae, Gh., Mahdavi, S.R., Mohammadi Nokhandani, A., Taheri Otaghsara, M. and Khadem Abolfazli, M. (2015) Effect of Mega Voltage Energy on Dose Enhancement in Phantom Study by Using Gold Nanoparticle Polymer Gel Dosimeter. International Journal of Biomedical Science and Engineering, 3, 1-4. http://dx.doi.org/10.11648/j.ijbse.20150301.11 